\title{
Coinfection of Helicobacter pylori and Hepatitis C Virus in the Development of Hepatocellular Carcinoma: A Systematic Review and Meta-Analysis
}

\author{
Samragnyi Madala ${ }^{a}$, Kira MacDougall ${ }^{\mathrm{b}}$, Balarama Krishna Surapanenic, Robin Park ${ }^{\mathrm{d}}$, \\ Mohit Girotra ${ }^{\mathrm{e}}$, Anup Kasi ${ }^{\mathrm{f}} \mathrm{g}$
}

\begin{abstract}
Background: The relationship between Helicobacter pylori (H. pylori) and hepatocellular carcinoma (HCC) was firstly proposed in 1994 after Ward et al demonstrated the role of Helicobacter hepaticus in the development of HCC in mice. Studies also investigated the role of hepatitis B virus (HBV) and hepatitis $\mathrm{C}$ virus (HCV) coexisting with $H$. pylori in causing HCC. A causal relationship was never confirmed, and the relationship remains controversial. This meta-analysis aimed to summarize the research on this topic and investigate if a relationship exists between $H$. pylori infection and the development of $\mathrm{HCC}$ and if the presence of $\mathrm{HCV}$ and $\mathrm{HBV}$ along with $\mathrm{H}$. pylori plays a role in liver carcinogenesis.
\end{abstract}

Methods: Following PRISMA guidelines, we performed a systematic review of all relevant studies published in the literature using the keywords "Helicobacter pylori" and "hepatocellular carcinoma" on major literature databases, including PubMed, EMBASE, Web of Science, and Cochrane controlled trials register. A total of 656 research studies were identified between 1994 and 2020. Of those, 26 qualified under our selection criteria. Patients who were positive for HCC were classified as cases and those who did not have HCC were classified as controls. The H. pylori status and HCV status, if available, were identified for both groups. Statistical analysis was carried out by a biostatistician according to the Cochrane reviewer's handbook.

Manuscript submitted November 12, 2021, accepted November 29, 2021 Published online December 28, 2021

aDepartment of Geriatric Medicine, University of Kansas Medical Center, Kansas City, KS, USA

bDivision of Medical Oncology, University of Oklahoma, Norman, OK, USA 'Department of Internal Medicine, Aventura Hospital and Medical Center, Aventura, FL, USA

dDepartment of Internal Medicine, MetroWest Medical Center, Framingham, MA, USA

eDivision of Gastroenterology and Therapeutic Endoscopy, Swedish Medical Center, Seattle, WA, USA

fDivision of Medical Oncology, University of Kansas Medical Center, Westwood, KS, USA

${ }^{g}$ Corresponding Author: Anup Kasi, Division of Medical Oncology, University of Kansas Medical Center, Westwood, KS, USA.

Email: anupdoc@gmail.com

doi: https://doi.org/10.14740/jocmr4637
Results: Out of the 26 studies included in the final analysis, 13 were retrospective case-control studies, 11 were cross-sectional studies, and two were prospective case-control and cohort studies. Overall, the prevalence of $H$. pylori infection was $64.78 \%$ (561 of 866) amongst HCC cases and $47.92 \%(1,718$ of 3,585$)$ in the non-HCC control group. The summary odds ratio (OR) for the association of H. pylori infection with the risk for HCC (using the random-effects model, which accounted for the heterogeneity across the 26 studies) was determined to be 4.75 (95\% confidence interval (CI): 3.06 - 7.37, $\left.\mathrm{I}^{2}=63 \%\right)$. We also performed a subgroup analysis to determine the odds of developing HCC in the presence of H. pylori and HCV coinfection. The summary OR of it was 12.76 (95\% CI: 4.13 - 39.41, $\mathrm{I}^{2}=78 \%$ ). The summary OR for the risk of developing HCC in the presence of $\mathrm{HCV}$ infection without $H$. pylori infection was $2.21(95 \%$ CI: $0.70-6.94, \mathrm{I}^{2}=79 \%$ ). Whereas, the odds of developing HCC in the presence of only $H$. pylori infection without $\mathrm{HCV}$ was found to be 0.54 (95\% CI: $\left.0.11-2.63, \mathrm{I}^{2}=80 \%\right)$. There was inconsistency in the data presented in some studies regarding $\mathrm{HCV}$ infection status. Since data were extracted from different study designs, subgroup analysis by study design was performed which showed no significant difference between the study groups $(\mathrm{P}=0.5705)$.

Conclusion: This meta-analysis demonstrates a positive association between $H$. pylori infection and the development of HCC. There is a significantly higher risk of developing $\mathrm{HCC}$ in the presence of $\mathrm{HCV}$ infection along with $H$. pylori. Further prospective cohort studies are needed to prove the causal relationship, especially in cases of HBV and HCV coinfection, and cirrhotic patients.

Keywords: Helicobacter pylori; Hepatitis C virus; Hepatocellular carcinoma; Systematic review; Meta-analysis

\section{Introduction}

In 1984, Helicobacter pylori (H. pylori) was firstly isolated by Nobel prize winners Marshall and Warren from gastric biopsy specimens of patients suffering from chronic gastritis and peptic ulcer disease [1]. H. pylori is a gram-negative spiral-shaped, microaerophilic, flagellated bacterium known to colonize gastric mucosa. This bacterium is believed to have originated in East Africa, and has been co-existent in the stomach of humans 
for at least 58,000 years [2]. Worldwide, the prevalence of $H$. pylori is estimated to be around $44.3 \%$ [3]. The bacterium can survive in the acidic environment of the stomach through the secretion of an enzyme called urease, which hydrolyzes urea to ammonia. This creates the alkaline medium necessary for survival and impairs the host's defense mechanisms [4]. Other colonizing mechanisms include using chemoreceptors to identify and swim to less acidic environments within the gastric mucosa. Huang et al demonstrated that bacteria lacking membrane bound chemoreceptors, called transducer-like proteins (TIp), TlpA, and TlpD are 100-fold less potent in colonizing and surviving the acidic environment of the stomach [5]. While most infected patients remain asymptomatic, chronic infections may lead to gastritis, peptic, and duodenal ulcers.

H. pylori was firstly described as a carcinogen in 1994, by the International Agency for Research on Cancer (IARC), and became the first known bacteria to cause cancer [6]. H. pylori has been linked to intestinal and diffuse-type gastric carcinoma and gastric mucosa-associated lymphoid tissue (MALT) lymphoma [7]. The bacteria is known to secrete the virulence factors cytotoxin-associated gene A (CagA) protein and vacuolating cytotoxin gene A ( VacA) protein which play an important role in carcinogenesis. CagA helps to invade the epithelial cells, and VacA induces apoptosis of the cells by activation of pro-apoptotic factor Bcl-2 associated X protein (Bax) [8]. After invasion of the epithelial cells, tumor cell proliferation occurs through inhibition of the activity of tumor suppressor gene, p53, thereby preventing apoptosis [9].

The same year $H$. pylori was deemed a carcinogen by the IARC, Helicobacter hepaticus (H. hepaticus) was found to cause hepatocellular carcinoma (HCC) in mice $[10,11]$. In humans, Helicobacter species such as Helicobacter bilis and Helicobacter pullorum have been isolated from the biliary duct and gallbladder in patients who underwent cholecystectomy for cholecystitis pointing towards a possible role of Helicobacter species in colonizing and possibly contributing to hepatobiliary diseases [12]. Enterohepatic circulation, reduction of bile $\mathrm{pH}$ through cholestasis creates a favorable environment for the growth of $H$. pylori in the liver and biliary tree [13]. In 2001, H. pylori was firstly cultured from a liver biopsy specimen collected from a patient with cirrhosis secondary to Wilson's disease [14]. Since then, several studies have investigated the role of helicobacter in causing chronic liver diseases, hepatitis and $\mathrm{HCC}$, either independently or through co-existing viral infections in the liver such as hepatitis B virus (HBV) and hepatitis $\mathrm{C}$ virus (HCV) [15]. A study done by Liu et al looked at the possibility of $H$. pylori in causing $\mathrm{HCC}$ through a histidine-rich cytoplasmic protein (hpn), which accounts for nearly $2 \%$ of total proteins synthesized by $H$. pylori. With nickel being a known carcinogen, hpn is a nickel-binding protein which acts by sequestering intracellular metal ions. Ubiquitin specific protease 5 (USP5) was identified as a molecular target of hpn protein. The study proved that hpn suppressed cell growth and induced apoptosis by suppressing USP5 expression in HCC cells [16]. Despite mounting evidence, the relationship between $H$. pylori and the development of HCC has not been proven in clinical studies, and hence, remains controversial. This systematic review and meta-analysis aimed to summarize all available research on this topic and investigate if a relation- ship exists between $H$. pylori infection and the development of $\mathrm{HCC}$, and if the presence of an HCV coinfection plays an important role in liver carcinogenesis.

\section{Materials and Methods}

\section{Protocol and registration}

The following study has not been registered in PROSPERO.

\section{Information sources}

Following preferred reporting items for systematic reviews and meta-analysis (PRISMA) guidelines, we performed a systematic search for Medical Subject Heading (MeSH) terms "Helicobacter pylori" and "hepatocellular carcinoma" on major literature search engines including PubMed, EMBASE, Web of science, and Cochrane controlled register of trials. We included studies that were published in both English and Chinese languages between 1994 and March 2020 that were relevant to our search criteria.

\section{Eligibility criteria}

\section{Inclusion criteria}

The following criteria were carefully applied in choosing the studies: studies performed only on human subjects were included. Case-control, cohort, and cross-sectional studies only were included in the meta-analysis. Cases and control groups must be clearly identified in the study. Cases should include confirmed diagnosis of HCC and controls should include patients without HCC. Cases should be diagnosed with HCC using a liver biopsy. Patients tested positive for H. pylori through standardized tests like fecal $H$. pylori antigen, urea breath test, polymerase chain reaction (PCR) testing from liver biopsy tissue, anti-H. pylori IgG antibody testing were included.

\section{Exclusion criteria}

Incomplete studies, case reports, letter to the editors, animal studies, and systematic reviews were excluded from the study. Studies on the relationship between $H$. pylori and cancers of the biliary tree were excluded. Patients diagnosed with cholangiocarcinoma within the included studies were excluded from our analysis.

\section{Data extraction}

Covidence, a software used to manage articles for systematic review and meta-analysis was used for data extraction. All articles were carefully reviewed by two independent researchers 
and screened using our pre-defined inclusion and exclusion criteria. Any discrepancies were thoroughly reviewed and resolved. Relevant data were collected from the studies including the name of the first author, year and type of the study design, sample size of cases and controls, baseline characteristics including mean age and socio-economic status where available, the detection method of H. pylori, and which liver pathology was present in both the cases and control groups.

\section{Risk of bias in individual studies}

Two independent reviewers used the Newcastle-Ottawa scale (NOS) to assess the quality of individual studies for case-control, cross-sectional and cohort studies. Total NOS score was calculated based on three categories: 1) selection; 2) comparability; and 3) exposure. The selection and exposure categories were in turn subdivided: selection was subdivided into: a) Is the case definition adequate; b) representation of cases; c) selection of controls; and d) definition of controls. Exposure is subdivided into: a) ascertainment of exposure; b) same method of ascertainment for cases and controls; and c) non-response rate. Each category is scored by a star based on the criteria met with a maximum score of nine stars.

\section{Statistical analysis}

The odds ratio (OR) was used as the summary measure for the incidence of HCC. Exposure was defined as H. pylori positivity. ORs were pooled using the inverse variance method. Random-effects model was applied using the DersimonianLaird estimator. Heterogeneity was estimated with the $\mathrm{I}^{2}$ value. Subgroup analyses were performed using: 1) "study design" for stratification; and 2) $H$. pylori and HCV coinfection as the exposure variable. The Begg funnel plot and Egger's test were used to assess small sample publication bias. A P-value of less than 0.05 indicates significant asymmetry and publication bias. Trim and fill analysis was applied to estimate the impact of publication bias on the effect size. Meta-analysis was performed using the package "meta" for the R project (ver. 4.0.0).

\section{Results}

\section{Characteristics of included studies}

A total of 656 publications were retrieved from the initial database search, and 390 studies were remaining after duplicates were removed. Following review by title and abstract, 276 studies were excluded because the articles were literature reviews, case reports, or studied biliary tract cancers as subjects. The remaining 114 studies were screened for full-text review, and after removal of 88 studies based on exclusion criteria, 26 publications were included in the final meta-analysis (Fig. 1). This process yielded 26 studies involving 866 cases (HCC) and 3,585 controls (non-HCC). Of these studies, 13 were retrospective case control, 11 were cross-sectional, and two were prospective case-control and cohort studies. The overall prevalence of $H$. pylori infection was $64.78 \%$ (561 of 866) in the cases and $47.92 \%(1,718$ of 3,585$)$ in the controls, respectively (Table 1). The mean age of the included studies ranged from 35.3 to 68 for cases and from 36.6 to 69 for controls (Table 2 [17-42]).

\section{Meta-analysis}

The primary outcome of our meta-analysis was the difference in H. pylori infection in HCC patients versus non-HCC patients. The pooled odds of $H$. pylori infection were significantly higher in cases (HCC) compared to controls (non-HCC) in the random-effects model (OR: 4.75, 3.06 - 7.37) for heterogeneity $\left(\mathrm{I}^{2}=63 \%\right)$ (Fig. 2).

Furthermore, subgroup analysis was conducted to evaluate the impact of differences in study design as well as $H$. pylori and HCV coinfection. First, subgroup analysis by study design showed no significant differences between the subgroups $(\mathrm{P}=$ $0.5705)$, suggesting that the differences in study design of the included studies did not systematically introduce bias. Second, the pooled odds of $H$. pylori and HCV coinfection was significantly higher in HCC patients versus non-HCC patients in the random-effects model (OR: 12.76, 4.13 - 39.41) for heterogeneity $\left(\mathrm{I}^{2}=78 \%\right.$ ) (Fig. 3 ). The pooled OR of this subgroup analysis was numerically higher than the meta-analysis of the primary outcome, and the difference between ORs was not statistically tested. Additionally, the pooled OR of $H$. pylori infection only in HCC patients was not significantly different compared to non-HCC patients in the random-effects model (OR: $0.54,0.11-2.63$ ) for heterogeneity $\left(\mathrm{I}^{2}=80 \%\right.$ ) (Fig. 4). The pooled OR of HCV infection only without $H$. pylori was non-significant (OR: 2.21, 0.70 - 6.94) for heterogeneity $\left(\mathrm{I}^{2}=\right.$ 79\%) (Fig. 5).

\section{Risk of bias across studies}

Begg's funnel plot (Fig. 6) and Egger's test showed significant asymmetry $(\mathrm{P}=0.00003)$ indicating significant small sample study bias. However, trim and fill analysis showed that results of the meta-analysis of the primary outcome remained significant after correction for small sample publication bias (OR: $2.79,1.79-4.34)$.

\section{Discussion}

This systematic review and meta-analysis included 24 retrospective and two prospective studies and investigated the relationship between $H$. pylori and the development of HCC. The results showed that there is an increased risk of developing $\mathrm{HCC}$ in the presence of $H$. pylori infection, and the risk is significantly higher with $\mathrm{HCV}$ and $H$. pylori coinfection. This is the first meta-analysis that also looked at the possibility of developing $\mathrm{HCC}$ in the presence of coinfection with $\mathrm{HCV}$. The results are especially relevant due to the increasing incidence 


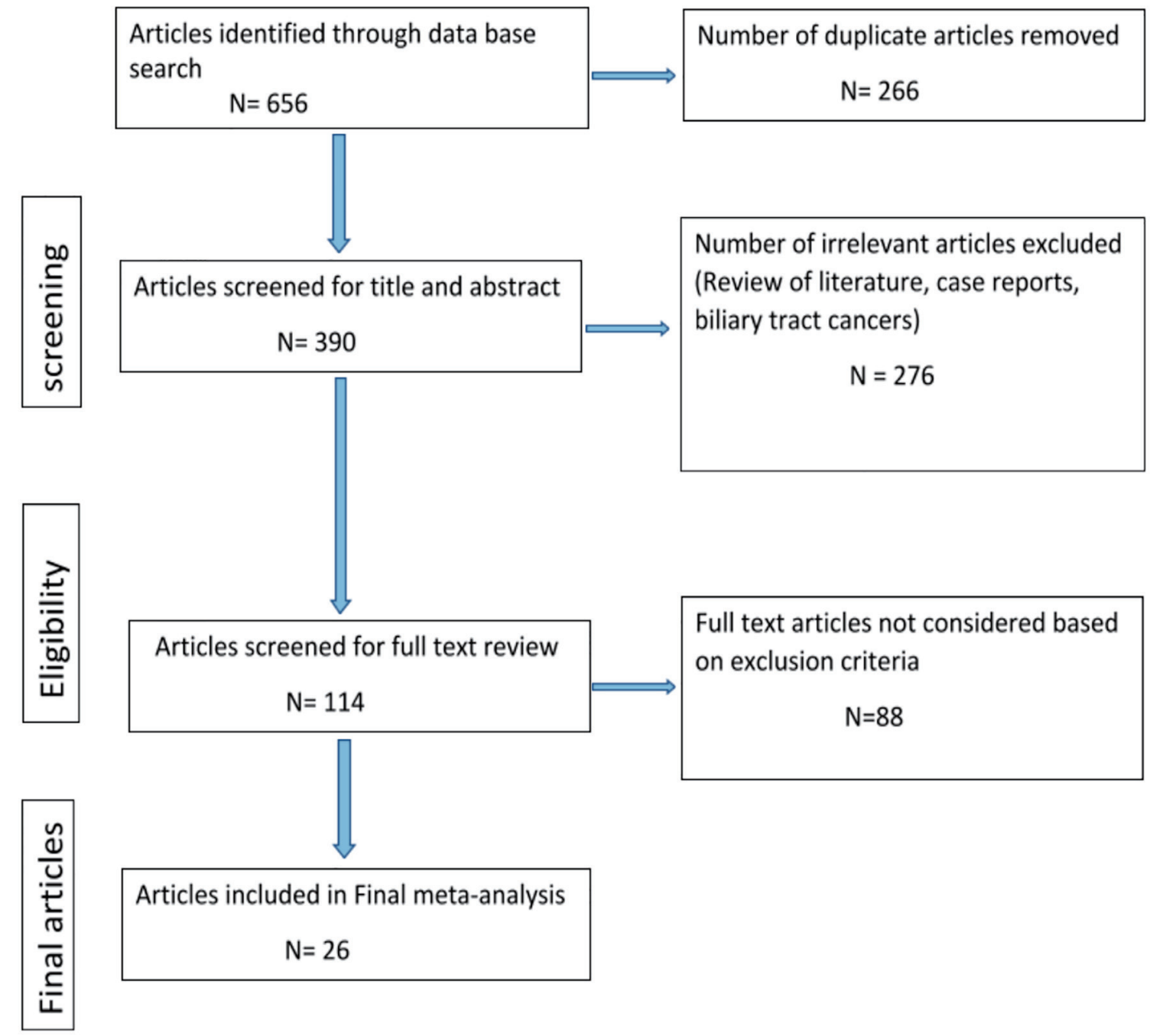

Figure 1. Flowchart of the articles selected from literature search.

of HCC. Over the last four decades, the incidence of HCC has almost tripled within the USA [43]. A 2018 worldwide analysis conducted by IARC reported the incidence of liver cancer to be as high as $4.7 \%$, with a mortality rate of $8.2 \%$, making this malignancy, the sixth most commonly diagnosed and the fourth leading cause of cancer-related deaths worldwide [44].
In 2018 alone, close to a million new cases $(840,000)$ of liver cancer were diagnosed with an estimated 782,000 deaths [44]. $\mathrm{HCC}$ is the most common primary liver cancer accounting for about $75-85 \%$ of liver cancers [45].

Most of the studies used PCR technique using primers from the $16 \mathrm{~S}$ ribosomal DNA to detect $H$. pylori. Growing $H$.

Table 1. Summary of the Results of the Primary and Subgroup Analysis

\begin{tabular}{llll}
\hline & HCC positive (cases) & HCC negative (controls) & $\begin{array}{l}\text { Random-effect odds ratio } \\
\text { (95\% confidence interval) }\end{array}$ \\
\hline $\begin{array}{l}\text { Primary analysis } \\
\quad \text { Relationship between H. pylori and HCC }\end{array}$ & $561 / 866(64.78 \%)$ & $1,718 / 3,585(47.92 \%)$ & $4.75(3.06-7.37)$ \\
Subgroup analysis & & & $12.76(4.13-39.41)$ \\
$\quad$ H. pylori and HCV coinfection & $168 / 422(39.81 \%)$ & $83 / 740(11.21 \%)$ & $0.54(0.11-2.63)$ \\
H. pylori infection only without HCV & $36 / 303(11.88 \%)$ & $79 / 495(15.95 \%)$ & $2.21(0.70-6.94)$ \\
\hline HCV only without H. pylori & $84 / 262(32.06 \%)$ & $141 / 485(29.07 \%)$ & \\
\hline
\end{tabular}

HCC: hepatocellular carcinoma; HCV: hepatitis C virus; H. pylori: Helicobacter pylori. 
Table 2. Characteristics of the Studies Included in the Meta-Analysis

\begin{tabular}{|c|c|c|c|c|c|c|}
\hline \multirow{2}{*}{ Study authors } & \multirow{2}{*}{$\begin{array}{l}\text { Year of } \\
\text { publication }\end{array}$} & \multirow{2}{*}{ Nature of the study } & \multicolumn{2}{|c|}{ H. pylori positive } & \multicolumn{2}{|c|}{ Mean age } \\
\hline & & & Cases & Controls & Cases & Controls \\
\hline Abdel-Razik et al [22] & 2020 & Prospective cohort & $16 / 23$ & $254 / 535$ & 55 & 55 \\
\hline Avenaud et al [27] & 2000 & Retrospective & $5 / 5$ & $1 / 8$ & 67.6 & 45.25 \\
\hline Coppola et al [23] & 2003 & Retrospective & $0 / 21$ & $0 / 34$ & 68 & 60.5 \\
\hline Fan et al [30] & 2002 & Retrospective & $9 / 15$ & $0 / 13$ & 48.5 & N/A \\
\hline Fotouhi et al [31] & 2011 & Retrospective & $7 / 22$ & $3 / 37$ & N/A & N/A \\
\hline Giannini et al [19] & 2003 & Retrospective & $23 / 33$ & $41 / 55$ & $64.6 \pm 9$ & 55 \\
\hline Qu et al [20] & 2017 & Case-control & $49 / 61$ & $813 / 1,521$ & $35.3 \pm 15.7$ & $36.6 \pm 14.5$ \\
\hline Leelawat et al [35] & 2007 & Retrospective & $12 / 12$ & $5 / 7$ & 54 & 59 \\
\hline Leone et al [21] & 2003 & Case-control & $36 / 46$ & $25 / 46$ & 60 & 69 \\
\hline Rocha et al [36] & 2005 & Cross sectional & $16 / 31$ & $18 / 78$ & 57.8 & 49 \\
\hline Mekonnen et al [37] & 2018 & Prospective case-control & $37 / 60$ & $14 / 60$ & 36 & 51 \\
\hline Murphy et al [38] & 2014 & Case-control & $91 / 97$ & $198 / 224$ & 58 & 57 \\
\hline Pellicano et al [25] & 2004 & Cross-sectional & $17 / 20$ & $2 / 6$ & N/A & N/A \\
\hline Verhoef et al [39] & 2003 & Cross-sectional & $9 / 20$ & $3 / 31$ & 61 & 62 \\
\hline Vivekanandan et al [24] & 2008 & Retrospective & $0 / 22$ & $5 / 60$ & $52.8 \pm 14$ & N/A \\
\hline Xuan et al [17] & 2006 & Cross-sectional & $17 / 28$ & $18 / 77$ & N/A & $\mathrm{N} / \mathrm{A}$ \\
\hline
\end{tabular}

H. pylori: Helicobacter pylori.

pylori in a culture is highly effective in detecting viable organisms but it is very challenging to isolate the bacteria from liver specimens due to inhibitory effect of bile acids. The biopsy specimens are usually stored at frozen temperatures up to -80 ${ }^{\circ} \mathrm{C}$ after surgical resection making them less viable [13]. Xuan and Yang et al of all the studies were able to use culture to grow and detect $H$. pylori $[17,18]$. Serology is relatively inexpensive, but due to the cross-reactivity between helicobacter and campylobacter, this can sometimes lead to false positive results. Using serology testing for $H$. pylori IgG antibody as in Giannini, Hao and Leone et al cannot differentiate between active versus chronic infection [19-21]. There is no gold standard method available to detect $H$. pylori in the liver. There needs to be histological evidence to prove the pathogenicity of $H$. pylori to argue if the finding of $H$. pylori was a result of enterohepatic circulation, transient colonization or colonization leading to liver diseases.

While most of the studies were retrospective and did not provide a causal relationship between $H$. pylori and HCC, the prospective study by Abdel-Razik et al was able to demonstrate a causal relationship on a large sample size of 558 cirrhotic patients [22]. All patients were tested for $H$. pylori using fecal antigen test. Out of the $270(48.5 \%)$ patients that were positive for H. pylori, 16 (5.9\%) developed HCC at 1-year follow-up. The results showed that not only does $H$. pylori play a role in the development of $\mathrm{HCC}$ with an incidence rate difference of 0.042 before and after treatment $(\mathrm{P}=0.037)$, it also increased the risk of cirrhotic complications, mainly portal vein thrombosis $(\mathrm{P}=0.043)$. The study also showed an increase in inflammatory factors such as serum $\mathrm{C}$-reactive protein, tumor necrosis factor- $\alpha$, interleukin- 6 , nitric oxide and vascular endothelial growth factor levels in patients infected with $H$. pylori. There was a decrease in the level of inflammatory markers and cirrhotic complications 1 year after treatment of $H$. pylori [22]. Since all the participants were tested for $H$. pylori at the beginning of the study and they were cirrhotic patients to begin 




Figure 2. Forest plot showing the risk of developing $\mathrm{HCC}$ in the presence of infection with $\mathrm{H}$. pylori. OR: odds ratio; $\mathrm{Cl}$ : confidence interval; HCC: hepatocellular carcinoma; H. pylori: Helicobacter pylori.

with, it is unclear for how long they remained infected with H. pylori.

Though there are many studies that showed positive results, Coppola and Vivekanandan et al had no patients positive for $H$. pylori in HCC group. Both were retrospective studies, used PCR analysis to test for H. pylori in liver biopsy samples using positive controls from gastric biopsy sections. Though the number of cases including both studies was only 43 out of $866(4.9 \%)$ cases included in the HCC group of our metaanalysis, the studies questioned the relationship that was being

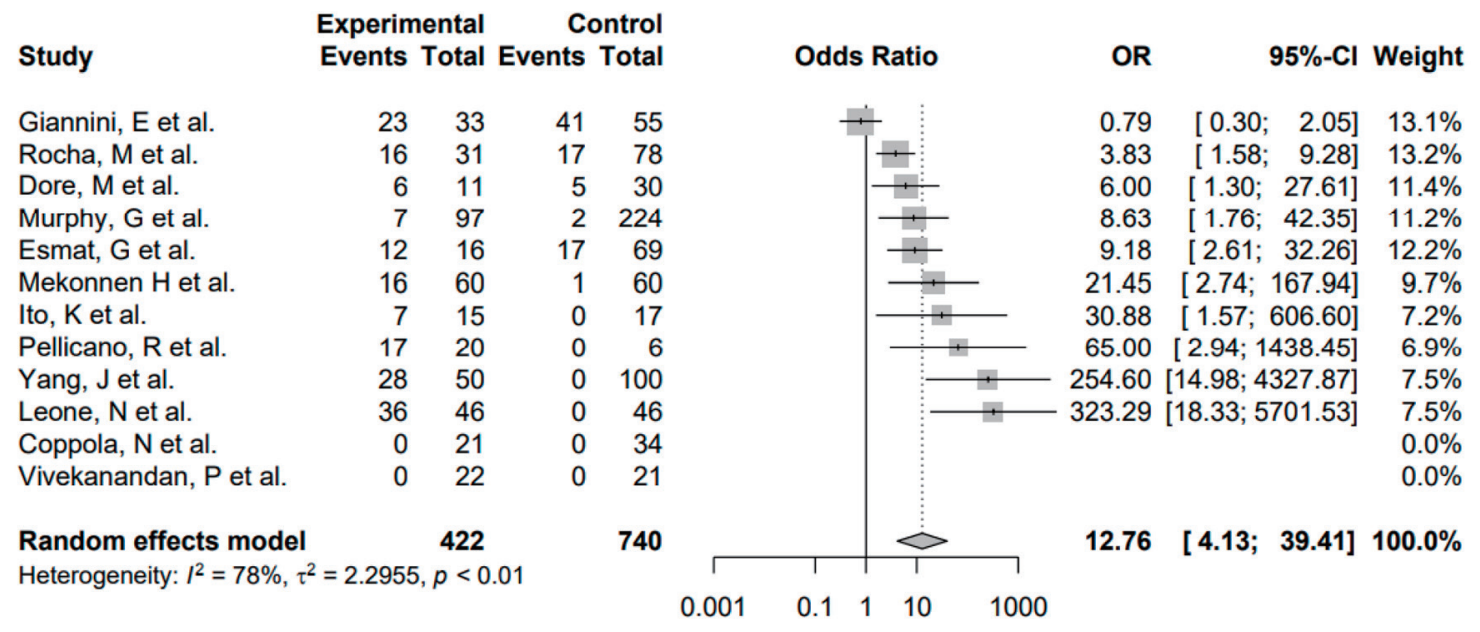

Figure 3. Forest plot showing the risk of developing $\mathrm{HCC}$ in the presence of $\mathrm{HCV}$ and $\mathrm{H}$. pylori coinfection. OR: odds ratio; $\mathrm{Cl}$ : confidence interval; HCC: hepatocellular carcinoma; HCV: hepatitis C virus; H. pylori: Helicobacter pylori. 


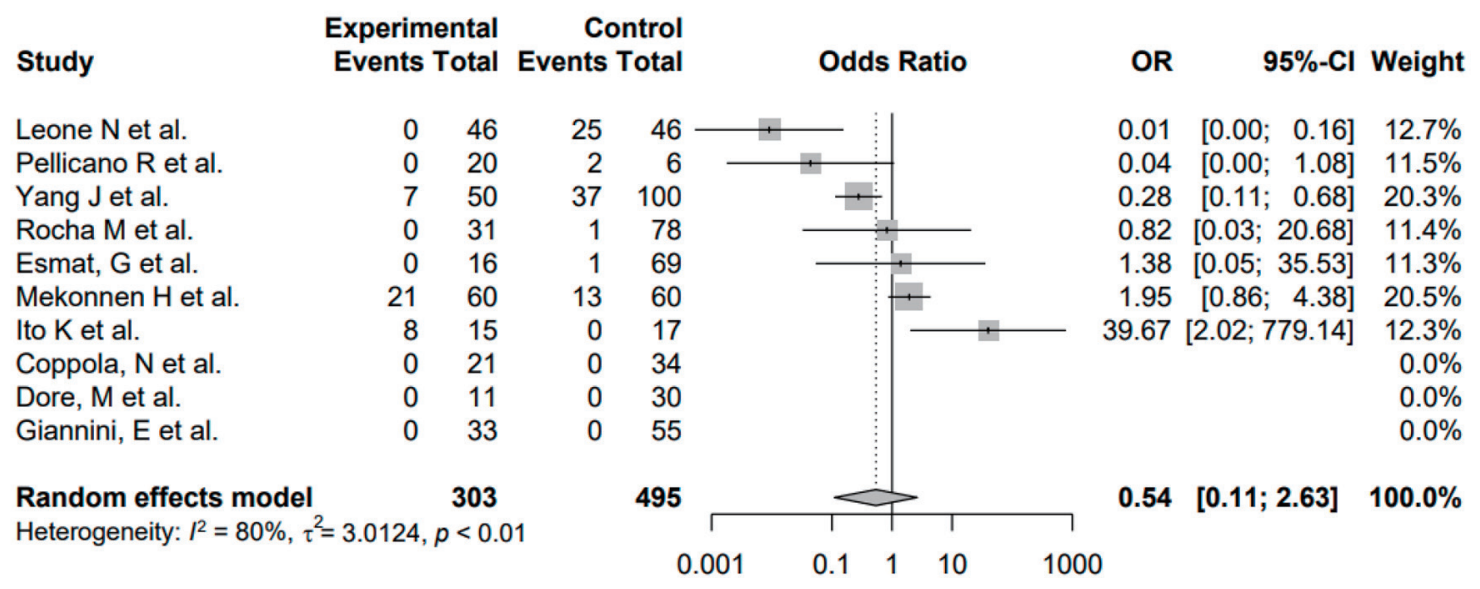

Figure 4. Forest plot showing the risk of developing $\mathrm{HCC}$ in the presence of $H$. pylori infection only without HCV. OR: odds ratio; $\mathrm{Cl}$ : confidence interval; HCC: hepatocellular carcinoma; HCV: hepatitis C virus; H. pylori: Helicobacter pylori.

investigated thus far $[23,24]$.

There were approximately 50,300 new infections of HCV in the USA in 2018, with an estimated 2.3 million people already living with the infection [46]. Overall, $50 \%$ of new liver cancer cases are secondary to HCV in the USA [47]. In view of the increasing prevalence of HCV infection, a subgroup analysis was performed to assess the possible role of $\mathrm{HCV}$ coinfection with $H$. pylori in the development of HCC. The presence of $\mathrm{H}$. pylori along with $\mathrm{HCV}$ can potentiate the development of HCC due to presence of chronic inflammation in the liver. Pellicano et al demonstrated $85 \%$ positivity in $\mathrm{H}$. pylori infection rates among patients that developed HCC secondary to HCVrelated cirrhosis [25]. Our subgroup analysis showed that the odds of developing $\mathrm{HCC}$ in the presence of $\mathrm{HCV}$ and $\mathrm{H}$. pylori infection is about 12.76. Fifteen out of the 26 studies included in the meta-analysis recorded the presence of $\mathrm{HCV}$ infection which provided us with a large sample size. The missing values in the data were adjusted for using a correction factor of 0.5 to minimize the error. Interestingly, $H$. pylori is known to be a strong initiator for inflammatory cascade, whereas $\mathrm{HCV}$ can create a chronic inflammatory state through secretion of interleukins $-1,-6$ and tumor necrosis factors, which together can help build a strong inflammatory state in the liver tissue potentiating the development of HCC [48].

With the recent use of direct antiviral therapy for treatment of HCV infection, there is hope for reduction of HCC incidence in these patients. However, several studies showed that there are still recurrences after achieving sustained virological response. The recurrences were mostly seen in patients with advanced fibrosis. Several prospective studies adjusted for risk factors like age, gender, presence of chronic medical conditions like diabetes, ischemic heart disease, renal failure, human immunodeficiency virus, and HBV but none of them looked at the incidence of $H$. pylori in this population [49, 50]. Based on our study results, there might be a possible role of H. pylori in causing $\mathrm{HCC}$ in these patients. If so, it is equally important to eradicate $H$. pylori along with HCV.

A similar meta-analysis which included 10 studies and 522 patients investigated the relationship between $H$. pylori and HCC in 2008. Like our meta-analysis, they were able to show a positive relationship with summary OR of 13.63. However, the study did not compare the relationship between $H$.

\section{Study}

Dore, $\mathrm{M}$ et al.

Esmat, $\mathrm{G}$ et al.

Rocha $\mathrm{M}$ et al.

Giannini, E et al.

Pellicano $R$ et al.

Coppola, $\mathrm{N}$ et al.

Leone $\mathrm{N}$ et al.

Vivekanandan $\mathrm{P}$ et al.

Yang $\mathrm{J}$ et al.

Leelawat $\mathrm{K}$ et al.

Random effects model

Heterogeneity: $I^{2}=79 \%, \tau^{2}=2.1512, p<0.01$

\section{Experimental Events Total Events Totrol}

$\begin{array}{rcrr}5 & 11 & 25 & 30 \\ 4 & 16 & 41 & 69 \\ 15 & 31 & 37 & 78 \\ 10 & 33 & 14 & 55 \\ 3 & 20 & 0 & 6 \\ 19 & 21 & 23 & 34 \\ 10 & 46 & 0 & 46 \\ 7 & 22 & 1 & 60 \\ 11 & 50 & 0 & 100 \\ 0 & 12 & 0 & 7 \\ & & & \\ 2.1512, p<0.01 & & \end{array}$

85

\section{Odds Ratio}

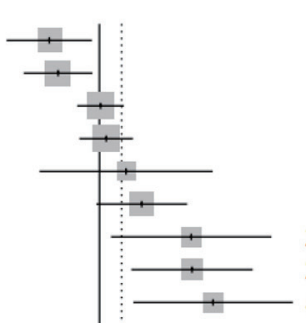

OR

95\%-Cl Weight

$0.17 \quad[0.04 ; 0.77] \quad 12.4 \%$

$0.23 \quad[0.07 ; 0.78] \quad 13.4 \%$

$1.04 \quad[0.45 ; 2.39] \quad 14.6 \%$

$1.27 \quad[0.49 ; 3.32] \quad 14.3 \%$

$2.60 \quad[0.12 ; 57.54] \quad 7.3 \%$

$4.54 \quad[0.90 ; 23.06] \quad 12.0 \%$

$26.75[1.52 ; 471.83] \quad 7.9 \%$

$27.53[3.14 ; 241.29] \quad 10.1 \%$

$58.52[3.37 ; 1016.99] \quad 8.0 \%$

$2.21[0.70 ; 6.94] 100.0 \%$

Figure 5. Forest plot showing the risk of developing $\mathrm{HCC}$ in the presence of $\mathrm{HCV}$ infection without $\mathrm{H}$. pylori. OR: odds ratio; $\mathrm{Cl}$ : confidence interval; HCC: hepatocellular carcinoma; HCV: hepatitis C virus; H. pylori: Helicobacter pylori. 


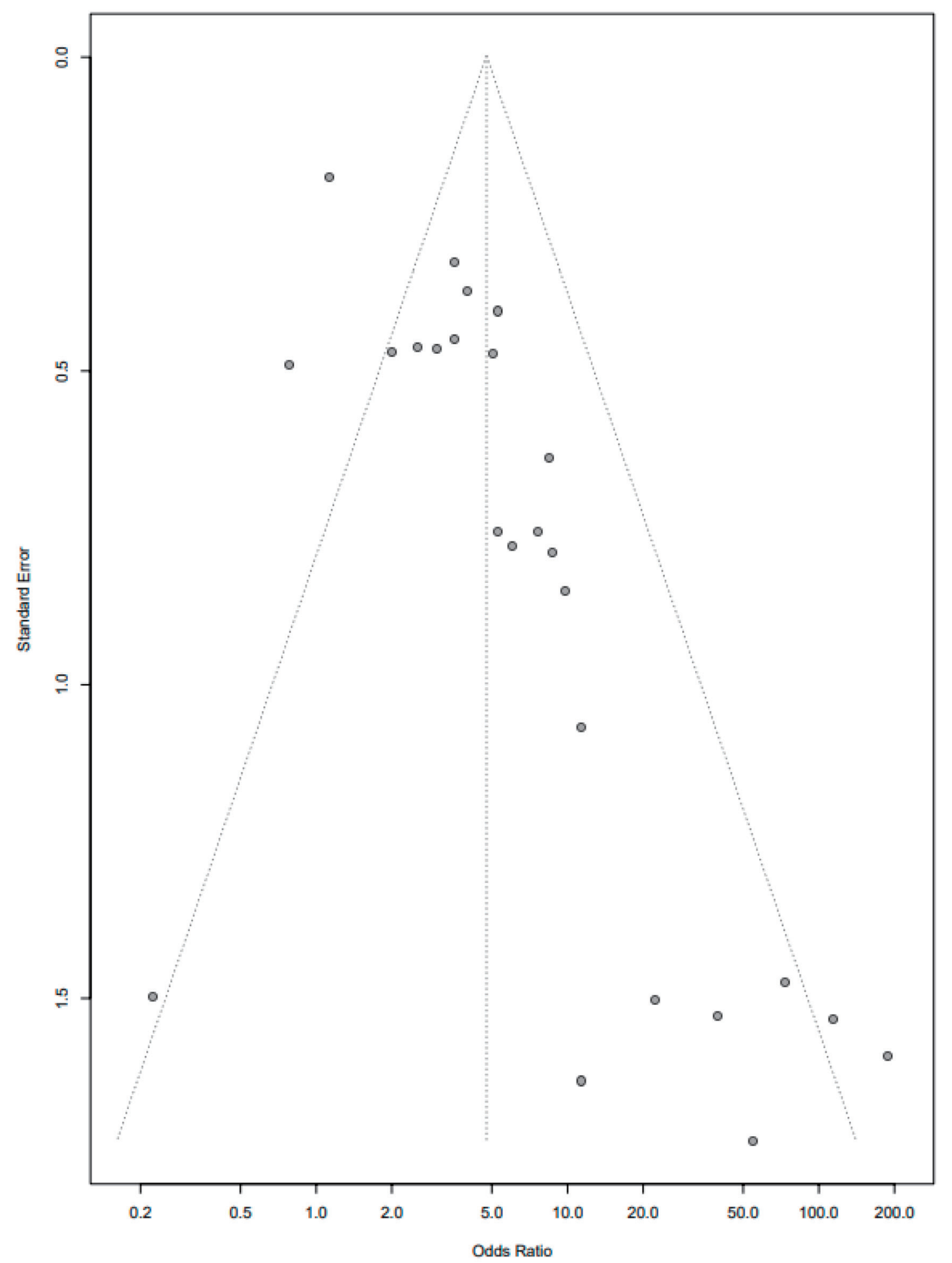

Figure 6. Funnel plot showing bias in selected studies.

pylori and $\mathrm{HCC}$ in association with $\mathrm{HCV}$ infection.

This meta-analysis has limitations. Firstly, a subgroup analysis was not performed on the HBV data as less than $25 \%$ of the studies provided information regarding HBV infection status. Though most of the studies are retrospective, there is some inconsistency in the design of the studies with presence of cirrhotic patients in both cases and control groups. There were no follow-up studies that tested for the results of HCC 
after eradication of $H$. pylori. $H$. pylori diagnoses were even considered if they had a positive IgG which may not necessarily mean active $H$. pylori infection in some of the cases. Future research should be directed at addressing these questions.

\section{Conclusion}

There is an increased risk of developing HCC in the presence of $H$. pylori infection, and that risk is significantly higher with $\mathrm{HCV}$ and H. pylori coinfection. Since both H. pylori and HCV are curable infections, treatment of these infections could possibly reduce the risk of developing HCC. The role of infectious agents in the development of HCC provides new insight on the pathogenesis of this disease and may influence screening recommendations in the future.

\section{Acknowledgments}

None to declare.

\section{Financial Disclosure}

Authors have no funding sources to disclose for the study.

\section{Conflict of Interest}

None to declare.

\section{Informed Consent}

Not applicable.

\section{Author Contributions}

Samragnyi Madala drafted the study, gathered data and wrote the manuscript. Kira Macdougall gathered data and edited the manuscript. Balarama K. Surapaneni edited the manuscript. Robin Park was statistician for the study. Anup Kasi drafted the article and revised it critically. Mohit Girotra approved the version to be published.

\section{Data Availability}

The processed data are available from the corresponding author upon request.

\section{References}

1. Marshall BJ, Warren JR. Unidentified curved bacilli in the stomach of patients with gastritis and peptic ulcera- tion. Lancet. 1984;1(8390):1311-1315.

2. Linz B, Balloux F, Moodley Y, Manica A, Liu H, Roumagnac P, Falush D, et al. An African origin for the intimate association between humans and Helicobacter pylori. Nature. 2007;445(7130):915-918.

3. Zamani M, Ebrahimtabar F, Zamani V, Miller WH, Alizadeh-Navaei R, Shokri-Shirvani J, Derakhshan MH. Systematic review with meta-analysis: the worldwide prevalence of Helicobacter pylori infection. Aliment Pharmacol Ther. 2018;47(7):868-876.

4. Marshall BJ, Barrett LJ, Prakash C, McCallum RW, Guerrant RL. Urea protects Helicobacter (Campylobacter) pylori from the bactericidal effect of acid. Gastroenterology. 1990;99(3):697-702.

5. Huang JY, Goers Sweeney E, Guillemin K, Amieva MR. Multiple acid sensors control Helicobacter pylori colonization of the stomach. PLoS Pathog. 2017;13(1):e1006118.

6. Schistosomes, liver flukes and Helicobacter pylori. IARC Working Group on the evaluation of carcinogenic risks to humans. Lyon, 7-14 June 1994. IARC Monogr Eval Carcinog Risks Hum. 1994;61:1-241.

7. Uemura N, Okamoto S, Yamamoto S, Matsumura N, Yamaguchi S, Yamakido M, Taniyama K, et al. Helicobacter pylori infection and the development of gastric cancer. N Engl J Med. 2001;345(11):784-789.

8. Nejati S, Karkhah A, Darvish H, Validi M, Ebrahimpour $\mathrm{S}$, Nouri HR. Influence of Helicobacter pylori virulence factors CagA and VacA on pathogenesis of gastrointestinal disorders. Microb Pathog. 2018;117:43-48.

9. Holmes L, Jr., Rios J, Berice B, Benson J, Bafford N, Parson K, Halloran D. Predictive effect of Helicobacter pylori in gastric carcinoma development: systematic review and quantitative evidence synthesis. Medicines (Basel). 2021;8(1):1.

10. Ward JM, Anver MR, Haines DC, Benveniste RE. Chronic active hepatitis in mice caused by Helicobacter hepaticus. Am J Pathol. 1994;145(4):959-968.

11. Fox JG, Dewhirst FE, Tully JG, Paster BJ, Yan L, Taylor NS, Collins MJ, Jr., et al. Helicobacter hepaticus sp. nov., a microaerophilic bacterium isolated from livers and intestinal mucosal scrapings from mice. J Clin Microbiol. 1994;32(5):1238-1245.

12. Fox JG, Dewhirst FE, Shen Z, Feng Y, Taylor NS, Paster BJ, Ericson RL, et al. Hepatic Helicobacter species identified in bile and gallbladder tissue from Chileans with chronic cholecystitis. Gastroenterology. 1998;114(4):755763.

13. Leong RW, Sung JJ. Review article: Helicobacter species and hepatobiliary diseases. Aliment Pharmacol Ther. 2002;16(6):1037-1045.

14. de Magalhaes Queiroz DM, Santos A. Isolation of a Helicobacter strain from the human liver. Gastroenterology. 2001;121(4):1023-1024.

15. Pellicano R, Menard A, Rizzetto M, Megraud F. Helicobacter species and liver diseases: association or causation? Lancet Infect Dis. 2008;8(4):254-260.

16. Liu Y, Wang WM, Zou LY, Li L, Feng L, Pan MZ, Lv MY, et al. Ubiquitin specific peptidase 5 mediates Histidinerich protein Hpn induced cell apoptosis in hepatocellu- 
lar carcinoma through P14-P53 signaling. Proteomics. 2017;17(12):1600350.

17. Xuan SY, Li N, Qiang X, Zhou RR, Shi YX, Jiang WJ. Helicobacter infection in hepatocellular carcinoma tissue. World J Gastroenterol. 2006;12(15):2335-2340.

18. Yang J, Ji S, Zhang Y, Wang J. Helicobacter hepaticus infection in primary hepatocellular carcinoma tissue. Singapore Med J. 2013;54(8):451-457.

19. Giannini E, Fasoli A, Botta F, Romagnoli P, Malfatti F, Chiarbonello B, Mamone M, et al. Helicobacter pylori infection is associated with greater impairment of cytochrome P-450 liver metabolic activity in anti-HCV positive cirrhotic patients. Dig Dis Sci. 2003;48(4):802-808.

20. Qu H. Helicobacter pylori morbidity in chronic hepatitis B patients: a case-control study. In: Sun Y, editor. Allied academics, Biomedical research; 2017.

21. Leone N, Pellicano R, Brunello F, Cutufia MA, Berrutti $\mathrm{M}$, Fagoonee $\mathrm{S}$, Rizzetto $\mathrm{M}$, et al. Helicobacter pylori seroprevalence in patients with cirrhosis of the liver and hepatocellular carcinoma. Cancer Detect Prev. 2003;27(6):494-497.

22. Abdel-Razik A, Mousa N, Elhelaly R, Elzehery R, Hasan AS, Abdelsalam M, Seif AS, et al. Helicobacter pylori as an initiating factor of complications in patients with cirrhosis: a single-center observational study. Front Med (Lausanne). 2020;7:96.

23. Coppola N, De Stefano G, Marrocco C, Scarano F, Scolastico C, Tarantino L, Rossi G, et al. Helicobacter spp. and liver diseases. Infez Med. 2003;11(4):201-207.

24. Vivekanandan P, Torbenson M. Low frequency of Helicobacter DNA in benign and malignant liver tissues from Baltimore, United States. Hum Pathol. 2008;39(2):213216.

25. Pellicano R, Mazzaferro V, Grigioni WF, Cutufia MA, Fagoonee S, Silengo L, Rizzetto M, et al. Helicobacter species sequences in liver samples from patients with and without hepatocellular carcinoma. World J Gastroenterol. 2004;10(4):598-601.

26. Abu Al-Soud W, Stenram U, Ljungh A, Tranberg KG, Nilsson HO, Wadstrom T. DNA of Helicobacter spp. and common gut bacteria in primary liver carcinoma. Dig Liver Dis. 2008;40(2):126-131.

27. Avenaud P, Marais A, Monteiro L, Le Bail B, Bioulac Sage P, Balabaud C, Megraud F. Detection of Helicobacter species in the liver of patients with and without primary liver carcinoma. Cancer. 2000;89(7):1431-1439.

28. Dore MP, Realdi G, Mura D, Graham DY, Sepulveda AR. Helicobacter infection in patients with HCV-related chronic hepatitis, cirrhosis, and hepatocellular carcinoma. Dig Dis Sci. 2002;47(7):1638-1643.

29. Esmat G, El-Bendary M, Zakarya S, Ela MA, Zalata K. Role of Helicobacter pylori in patients with HCV-related chronic hepatitis and cirrhosis with or without hepatocellular carcinoma: possible association with disease progression. J Viral Hepat. 2012;19(7):473-479.

30. Fan XG, Peng XN, Huang Y, Yakoob J, Wang ZM, Chen YP. Helicobacter species ribosomal DNA recovered from the liver tissue of chinese patients with primary hepatocellular carcinoma. Clin Infect Dis. 2002;35(12):1555-
1557.

31. Fotouhi M, Eslami G, Peirovi H, Hashemibahramani M, Douraghi M, Ahmadzadeh E. Survey of association between helicobacter pylori and hepatocellular carcinoma in the specimens derived from health centers of Shahid Beheshti University. Arch Clin Infect Dis. 2011;6(1):31-34.

32. Huang J, Cui J. Evaluation of Helicobacter pylori infection in patients with chronic hepatic disease. Chin Med J (Engl). 2017;130(2):149-154.

33. Huang Y, Fan XG, Wang ZM, Zhou JH, Tian XF, Li N. Identification of helicobacter species in human liver samples from patients with primary hepatocellular carcinoma. J Clin Pathol. 2004;57(12):1273-1277.

34. Ito K, Nakamura M, Toda G, Negishi M, Torii A, Ohno T. Potential role of Helicobacter pylori in hepatocarcinogenesis. Int J Mol Med. 2004;13(2):221-227.

35. Leelawat K, Suksumek N, Leelawat S, Lek-Uthai U. Detection of VacA gene specific for Helicobactor pylori in hepatocellular carcinoma and cholangiocarcinoma specimens of Thai patients. Southeast Asian J Trop Med Public Health. 2007;38(5):881-885.

36. Rocha M, Avenaud P, Menard A, Le Bail B, Balabaud C, Bioulac-Sage P, de Magalhaes Queiroz DM, et al. Association of Helicobacter species with hepatitis C cirrhosis with or without hepatocellular carcinoma. Gut. 2005;54(3):396-401.

37. Mekonnen HD, Fisseha H, Getinet T, Tekle F, Galle PR. Helicobacter pylori infection as a risk factor for hepatocellular carcinoma: a case-control study in ethiopia. Int J Hepatol. 2018;2018:1941728.

38. Murphy G, Michel A, Taylor PR, Albanes D, Weinstein SJ, Virtamo J, Parisi D, et al. Association of seropositivity to Helicobacter species and biliary tract cancer in the ATBC study. Hepatology. 2014;60(6):1963-1971.

39. Verhoef C, Pot RG, de Man RA, Zondervan PE, Kuipers EJ, JN IJ, Kusters JG. Detection of identical Helicobacter DNA in the stomach and in the non-cirrhotic liver of patients with hepatocellular carcinoma. Eur J Gastroenterol Hepatol. 2003;15(11):1171-1174.

40. Nilsson HO, Mulchandani R, Tranberg KG, Stenram U, Wadstrom T. Helicobacter species identified in liver from patients with cholangiocarcinoma and hepatocellular carcinoma. Gastroenterology. 2001;120(1):323-324.

41. Zhang SQ. The correlation between Helicob acter infection and hepatocellular carcinoma. Zhong Guo Zhongliu Linchuang. 2004;31:761-764.

42. Li N, Zhang SH, Xuan SY, Qiang X. [Study on Helicobacter infection in liver tissue from hepatocellular carcinoma]. Zhonghua Liu Xing Bing Xue Za Zhi. 2006;27(10):894-896.

43. Liver Cancer: Statistics. Cancer.net; 2020.

44. Bray F, Ferlay J, Soerjomataram I, Siegel RL, Torre LA, Jemal A. Global cancer statistics 2018: GLOBOCAN estimates of incidence and mortality worldwide for 36 cancers in 185 countries. CA Cancer J Clin. 2018;68(6):394424.

45. Rawla P, Sunkara T, Muralidharan P, Raj JP. Update in global trends and aetiology of hepatocellular carcinoma. Contemp Oncol (Pozn). 2018;22(3):141-150. 
46. Hepatitis C. Questions and answers for health professionals. Accessed Jun 3, 2021. https://www.cdc.gov/hepatitis/ hcv/hcvfaq.htm\#section1.

47. What is viral hepatitis? Centers for disease control and prevention. Accessed February 9, 2021. https://www.cdc. gov/hepatitis/abc/index.htm.

48. El-Masry S, El-Shahat M, Badra G, Aboel-Nour MF, Lotfy M. Helicobacter pylori and hepatitis $C$ virus coinfection in Egyptian patients. J Glob Infect Dis. 2010;2(1):49.
49. Pinero F, Mendizabal M, Ridruejo E, Herz Wolff F, Ameigeiras B, Anders M, Schinoni MI, et al. Treatment with direct-acting antivirals for HCV decreases but does not eliminate the risk of hepatocellular carcinoma. Liver Int. 2019;39(6):1033-1043.

50. Calvaruso V, Cabibbo G, Cacciola I, Petta S, Madonia S, Bellia A, Tine F, et al. Incidence of hepatocellular carcinoma in patients with $\mathrm{HCV}$-associated cirrhosis treated with direct-acting antiviral agents. Gastroenterology. 2018;155(2):411-421.e414. 